\title{
A family of half-transitive graphs
}

\author{
Jing Chen* \\ Department of Mathematics \\ Hunan First Normal University \\ Changsha, P. R. China \\ chenjing827@126.com
}

\author{
Cai Heng Li \\ School of Mathematics and Statistics \\ Yunnan University \\ Kunming, P. R. China \\ cai.heng.li@uwa.edu.au
}

\author{
Ákos Seress \\ School of Mathematics and Statistics \\ The Ohio State University \\ The University of Western Australia \\ Crawley, WA 6009, Australia
}

Submitted: Dec 15, 2011; Accepted: Mar 1, 2013; Published: Mar 8, 2013

Mathematics Subject Classifications: 05C25, 20B25

\begin{abstract}
We construct an infinite family of half-transitive graphs, which contains infinitely many Cayley graphs, and infinitely many non-Cayley graphs.
\end{abstract}

Keywords: half-transitive graphs; quotient graphs; automorphism groups.

\section{Introduction}

Let $\Gamma=(V, E)$ be a graph with vertex set $V$ and edge set $E$. A permutation of $V$ which preserves the adjacency of $\Gamma$ is an automorphism of the graph, and all automorphisms form the automorphism group Aut $\Gamma$. If a subgroup $G \leqslant \operatorname{Aut} \Gamma$ is transitive on $V$ or $E$, then $\Gamma$ is called $G$-vertex-transitive or $G$-edge-transitive, respectively. An ordered pair of adjacent vertices is called an arc, and $\Gamma$ is called arc-transitive if Aut $\Gamma$ is transitive on the set of arcs. An arc-transitive graph is vertex-transitive and edge-transitive, but the converse statement is not true. A graph which is vertex-transitive and edge-transitive but not arc-transitive is called half-transitive.

The study of half-transitive graphs was initiated with a question of Tutte [20, p. 60] regarding their existence, and he proved that a vertex-transitive and edge-transitive graph

${ }^{*}$ This work was partially supported by an ARC Discovery Project Grant, NNSF of China(No. 11171292, 11271208) and Hunan First Normal University(No. XYS12N12). 
with odd valency must be arc-transitive. Bouwer [4] in 1970 constructed the first family of half-transitive graphs. Since then, constructing and characterizing half-transitive graphs has been an active topic in algebraic graph theory, refer to $[1,2,10,13,16,22]$ and a survey [12] for the work during 1990's, and [14, 16, 17, 18, 19] for more recent work.

In this paper, we present an infinite family of half-transitive graphs. These graphs were originated from Johnson graphs $\mathbf{J}(n, i)$ where $i=1,2$ or 3 , the vertex set of which consists of the $i$-element subsets of an $n$-element set such that two vertices are adjacent when they meet in $(i-1)$-elements. It is known that the automorphism group of $\mathbf{J}(n, i)$ is $\mathrm{S}_{n}$, see [9] or [15, Theorem 1].

As usual, we denote by $[n]$ the set $\{1,2,3, \ldots, n\}$. Let

$$
V_{n}=\{\{\{i, j\}, k\} \mid i, j, k \in[n]\} .
$$

For convenience, we simply write the vertex $\{\{i, j\}, k\}$ as $(i j, k)$. Then $(i j, k)=(j i, k)$, and a 3 -subset $\{i, j, k\}$ corresponds to exactly three vertices $(i j, k),(i k, j)$ and $(j k, i)$. Thus, the cardinality is

$$
\left|V_{n}\right|=3\left(\begin{array}{l}
n \\
3
\end{array}\right)=n(n-1)(n-2) / 2 .
$$

Definition 1. For an integer $n>3$, let $\Gamma_{n}$ be the graph with vertex set $V_{n}$ such that two vertices $(i j, k)$ and $\left(i^{\prime} j^{\prime}, k^{\prime}\right) \in V_{n}$ are adjacent if and only if

$$
\{i, j\}=\left\{i^{\prime}, k^{\prime}\right\} \text { or }\left\{j^{\prime}, k^{\prime}\right\}
$$

and $\{i, j, k\} \neq\left\{i^{\prime}, j^{\prime}, k^{\prime}\right\}$.

The graph $\Gamma_{n}$ is regular and has valency $4(n-3)$. For example, the vertex $(12,3)$ has neighborhood

$$
\{(1 i, 2),(2 i, 1),(13, i),(23, i) \mid i>3\}
$$

which has size $4(n-3)$.

Let $\Gamma=(V, E)$ be a graph, and let $\mathcal{B}$ be a partition of the vertex set $V$. Then the quotient graph $\Gamma_{\mathcal{B}}$ induced on $\mathcal{B}$ is the graph with vertex set $\mathcal{B}$ such that $B, B^{\prime}$ are adjacent if and only if there is an edge which lies between $B$ and $B^{\prime}$. In this case, $\Gamma$ is also said to be homomorphic to $\Gamma_{\mathcal{B}}$.

A graph $\Gamma=(V, E)$ is called a Cayley graph if there is a group $R$ and a self-inversed subset $S \subset R$ such that $V=R$ and $u, v \in S$ are adjacent if and only if $v u^{-1} \in S$. Cayley graphs are vertex-transitive, but a vertex-transitive graph is not necessarily a Cayley graph. For example, the Petersen graph is the smallest vertex-transitive graph which is not a Cayley graph. The family of graphs $\Gamma_{n}$ contains infinitely many non-Cayley graphs.

Theorem 2. Let $\Gamma_{n}$ be a graph defined above. Then the following statements hold:

(i) $\Gamma_{n}$ is of order $\frac{n(n-1)(n-2)}{2}$, valency $4(n-3)$, girth 3 , and diameter 3 ;

(ii) $\Gamma_{n}$ is homomorphic to $\mathbf{K}_{n}, \mathbf{J}(n, 2)$ and $\mathbf{J}(n, 3)$; 
(iii) $\Gamma_{4}$ and $\Gamma_{5}$ are arc-transitive, and for $n \geqslant 6$, Aut $\Gamma_{n}=\operatorname{Sym}([n])$, and $\Gamma_{n}$ is halftransitive;

(iv) $\Gamma_{n}$ is a Cayley graph if and only if $n=8$, or $n=q+1$ with $q$ a prime-power, and $q \equiv 3(\bmod 4)$.

\section{Edge-transitivity}

Let $\sigma \in \operatorname{Sym}([n])$. For convenience, we simply denote $V_{n}$ by $V$, and denote $\Gamma_{n}$ by $\Gamma$. Then $\sigma$ induces a permutation on the vertex set $V$. Since $G=\operatorname{Sym}([n])$ is 3-transitive on $[n], G$ is transitive on $V$.

Take an edge $\left\{\left(i_{1} j_{1}, k_{1}\right),\left(i_{2} j_{2}, k_{2}\right)\right\}$ of $\Gamma$. Then $\left\{i_{1}, j_{1}\right\}=\left\{i_{2}, k_{2}\right\}$ or $\left\{j_{2}, k_{2}\right\}$, and hence $\left\{i_{1}^{\sigma}, j_{1}^{\sigma}\right\}=\left\{i_{2}^{\sigma}, k_{2}^{\sigma}\right\}$ or $\left\{j_{2}^{\sigma}, k_{2}^{\sigma}\right\}$. Thus, $\left(i_{1}^{\sigma} j_{1}^{\sigma}, k_{1}^{\sigma}\right)$ and $\left(i_{2}^{\sigma} j_{2}^{\sigma}, k_{2}^{\sigma}\right)$ are adjacent, that is, $\sigma$ maps edges to edges. Similarly, $\sigma$ maps non-edges to non-edges. So $\sigma$ is an automorphism of $\Gamma$, and $G=\operatorname{Sym}([n])$ is a vertex-transitive automorphism group of $\Gamma$.

Lemma 3. The graph $\Gamma=\Gamma_{n}$ is G-vertex-transitive and $G$-edge-transitive, but not $G$ arc-transitive.

Proof. We consider the edges incident with the vertex $\alpha=(12,3)$. The stabilizer $G_{\alpha}=$ $\operatorname{Sym}(\{1,2\}) \times \operatorname{Sym}(\{4,5, \ldots, n\}) \cong \mathrm{S}_{2} \times \mathrm{S}_{n-3}$, and $G_{\alpha}$ acting on the neighborhood $\Gamma(\alpha)$ has two orbits $\{(1 i, 2),(2 i, 1) \mid i>3\}$ and $\{(13, i),(23, i) \mid i>3\}$. Thus, $G$ is not transitive on the arcs of $\Gamma$. Further, the element $g=(23 i)$ maps $(1 i, 2)$ to $(12,3)$, and $(12,3)$ to $(13, i)$, so $g$ maps the edge $\{(1 i, 2),(12,3)\}$ to the edge $\{(12,3),(13, i)\}$. Since $\Gamma$ is $G$-vertex-transitive, we conclude that $\Gamma$ is $G$-edge-transitive.

Let $H$ be a subgroup of $G$, and $S$ be a subset of $G$. Define the coset graph of $G$ with respect to $H$ and $S$ to be the directed graph with vertex set $[G: H]$ and such that, for any $H x, H y \in V, H x$ is connected to $H y$ if and only if $y x^{-1} \in H S H$ and denote the digraph by $\operatorname{Cos}(G, H, H S H)$. With the vertex $\alpha=(12,3) \in V_{n}$ and element $g=(234) \in G$, the graph $\Gamma=\Gamma_{n}$ can be described as a coset graph $\operatorname{Cos}\left(G, G_{\alpha}, G_{\alpha}\left\{g, g^{-1}\right\} G_{\alpha}\right)$, which has vertex set $\left[G: G_{\alpha}\right]=\left\{G_{\alpha} x \mid x \in G\right\}$ such that $G_{\alpha} x$ and $G_{\alpha} y$ are adjacent if and only if $y x^{-1} \in G_{\alpha}\left\{g, g^{-1}\right\} G_{\alpha}$. The right multiplication of each element $g \in G$

$$
g: \quad G_{\alpha} x \mapsto G_{\alpha} x g, \text { for all } x \in G
$$

induces an automorphism of $\Gamma$.

Lemma 4. If an automorphism $\tau \in \operatorname{Aut}(G)$ normalizes $G_{\alpha}$ and $\left(G_{\alpha}\left\{g, g^{-1}\right\} G_{\alpha}\right)^{\tau}=$ $G_{\alpha}\left\{g, g^{-1}\right\} G_{\alpha}$, then $\tau$ is an automorphism of $\Gamma$.

Proof. Since $\tau$ normalizes $G_{\alpha}$, it induces a permutation on the vertex set $\left[G: G_{\alpha}\right]$.

For any two vertices $G_{\alpha} x$ and $G_{\alpha} y$, we have

$$
\begin{aligned}
G_{\alpha} x \sim G_{\alpha} y & \Longleftrightarrow y x^{-1} \in G_{\alpha}\left\{g, g^{-1}\right\} G_{\alpha} \\
& \Longleftrightarrow\left(y x^{-1}\right)^{\tau} \in\left(G_{\alpha}\left\{g, g^{-1}\right\} G_{\alpha}\right)^{\tau} \\
& \Longleftrightarrow y^{\tau}\left(x^{\tau}\right)^{-1} \in G_{\alpha}\left\{g, g^{-1}\right\} G_{\alpha} \\
& \Longleftrightarrow\left(G_{\alpha} x\right)^{\tau}=G_{\alpha} x^{\tau} \sim G_{\alpha} y^{\tau}=\left(G_{\alpha} y\right)^{\tau} .
\end{aligned}
$$


Thus, $\tau$ is an automorphism of the graph $\Gamma$.

Lemma 5. Both $\Gamma_{4}$ and $\Gamma_{5}$ are arc-transitive.

Proof. Let $\alpha=(12,3)$, and $g=(234)$. Consider first the case $G=\mathrm{S}_{4}$. Then $G_{\alpha}=$ $\operatorname{Sym}(\{1,2\})$, and $\Gamma=\operatorname{Cos}\left(G, G_{\alpha}, G_{\alpha}\left\{g, g^{-1}\right\} G_{\alpha}\right)$. Let $\tau$ be the inner-automorphism induced by the element $(34) \in G$. Then $\tau$ normalizes $G_{\alpha}$ and $g^{\tau}=g^{-1}$. By Lemma $4, \tau$ is an automorphism of the graph. Further, for the edge $\left\{G_{\alpha}, G_{\alpha} g\right\}$, we have

$$
\left(G_{\alpha}, G_{\alpha} g\right)^{\tau g}=\left(G_{\alpha}, G_{\alpha} g^{-1}\right)^{g}=\left(G_{\alpha} g, G_{\alpha}\right),
$$

and so $\Gamma$ is arc-transitive.

Next, consider the case $G=\mathrm{S}_{5}$. Again let $\alpha=(12,3)$ and $g=(234)$. Then $G_{\alpha}=$ $\operatorname{Sym}(\{1,2\}) \times \operatorname{Sym}(\{4,5\})$, and $\Gamma=\operatorname{Cos}\left(G, G_{\alpha}, G_{\alpha}\left\{g, g^{-1}\right\} G_{\alpha}\right)$. Let $\tau$ be the innerautomorphism of $G$ induced by the element $(15)(24) \in G$. Then $\tau$ normalizes $G_{\alpha}$ and reverses $g$. Arguing as above shows that $\Gamma$ is arc-transitive.

However, we will show that $\Gamma_{n}$ for $n \geqslant 6$ are all half-transitive.

\section{The parameters}

Denote the graph $\Gamma_{n}$ simply by $\Gamma$ in the following. For vertices $\alpha=\left(i_{1} j_{1}, k_{1}\right)$ and $\beta=$ $\left(i_{2} j_{2}, k_{2}\right)$, we denote $\left\{i_{1}, j_{1}, k_{1}\right\} \cap\left\{i_{2}, j_{2}, k_{2}\right\}$ by $\alpha \cap \beta$. Then $|\alpha \cap \beta|=2$ if $\alpha$ and $\beta$ are adjacent.

Lemma 6. The graph $\Gamma=\Gamma_{n}$ is of order $\frac{n(n-1)(n-2)}{2}$, valency $4(n-3)$, girth 3 , and diameter 3 .

Proof. As noticed above, each 3-subset $\{i, j, k\}$ corresponds to exactly three vertices $(i j, k),(j k, i)$ and $(i k, j)$. Thus, the order $|V|$ of $\Gamma$ equals $3\left(\begin{array}{l}n \\ 3\end{array}\right)=\frac{n(n-1)(n-2)}{2}$. By the definition of the graph $\Gamma$, the neighborhood of the vertex $\alpha=(i j, k)$ is $\Gamma(\alpha)=$ $\{(i k, m),(j k, m),(i m, j),(j m, i) \mid m \neq i, j, k\}$. Thus, $\Gamma$ is of valency $4(n-3)$. Moreover, since $(j k, m)$ and $(j m, i)$ are adjacent, $\Gamma$ is of girth 3 .

We next compute the distance $d(\alpha, \beta)$ between two vertices $\alpha$ and $\beta$. As $\Gamma$ is a vertex-transitive graph, we take $\alpha=(12,3)$. We first consider a small case that $n=4$. Then $|V|=12$, and the neighborhood $\Gamma(\alpha)=\{(14,2),(24,1),(13,4),(23,4)\}$. For other vertices except $(12,4)$, we have

$$
\Gamma(\alpha) \cap \Gamma(\beta)= \begin{cases}\{(23,4)\}, & \text { if } \beta=(13,2), \\ \{(13,4)\}, & \text { if } \beta=(23,1), \\ \{(24,1)\}, & \text { if } \beta=(14,3), \\ \{(14,2),(23,4)\}, & \text { if } \beta=(34,1), \\ \{(14,2)\}, & \text { if } \beta=(24,3), \\ \{(24,1),(13,4)\}, & \text { if } \beta=(34,2),\end{cases}
$$

and so $d(\alpha, \beta)=2$. For $\beta=(12,4), \Gamma(\alpha) \cap \Gamma(\beta)=0$. Furthermore, the sequence

$$
\alpha=(12,3),(14,2),(24,3), \beta=(12,4)
$$


is a path between $\alpha$ and $\beta$ of length 3. Hence, $d(\alpha, \beta)=3$, and thus, the graph $\Gamma$ is of diameter 3.

Now we treat the cases where $n \geqslant 5$. Take $\alpha=(12,3)$, and let $\beta=(i j, k)$.

Case 1. Assume first that $i, j, k \geqslant 4$. If a vertex $\gamma=\left(i^{\prime} j^{\prime}, k^{\prime}\right)$ is adjacent to both $\alpha$ and $\beta$, then $|\gamma \cap \alpha|=2$ and $|\gamma \cap \beta|=2$, which is not possible. Thus, $d(\alpha, \beta)$ is at least 3. On the other hand, the sequence

$$
\alpha=(12,3),(1 i, 2),(i k, 1), \beta=(i j, k)
$$

is a path between $\alpha$ and $\beta$ of length 3. Hence, $d(\alpha, \beta)=3$.

Case 2. Next, consider the case where $|\alpha \cap \beta|=1$.

Suppose that $\beta=(i j, 3)$, where $i, j \geqslant 4$. Then the sequence $\alpha,(1 i, 2),(i 3,1), \beta$ is a path between $\alpha$ and $\beta$, and hence $d(\alpha, \beta) \leqslant 3$. As mentioned above,

$$
\Gamma(\alpha)=\left\{\left(1 i^{\prime}, 2\right),\left(2 i^{\prime}, 1\right),\left(13, i^{\prime}\right),\left(23, i^{\prime}\right) \mid i^{\prime}>3\right\},
$$

and similarly,

$$
\Gamma(\beta)=\left\{\left(i j^{\prime}, j\right),\left(j j^{\prime}, i\right),\left(3 i, j^{\prime}\right),\left(3 j, j^{\prime}\right) \mid j^{\prime} \notin\{3, i, j\}\right\} .
$$

Thus, $\Gamma(\alpha) \cap \Gamma(\beta)=\emptyset$, and so $d(\alpha, \beta)=3$.

Assume now that $k \neq 3$. Then $\beta=(1 i, k),(2 i, k),(3 i, k),(i j, 1)$ or $(i j, 2)$, where $i, j, k \geqslant 4$. In each of these cases, $d(\alpha, \beta)=2$ because

$$
\Gamma(\alpha) \cap \Gamma(\beta)= \begin{cases}\{(1 k, 2),(13, i),(2 i, 1)\}, & \text { if } \beta=(1 i, k), \\ \{(2 k, 1),(23, i),(1 i, 2)\}, & \text { if } \beta=(2 i, k), \\ \{(31, i),(32, i)\}, & \text { if } \beta=(3 i, k), \\ \{(1 i, 2),(1 j, 2)\}, & \text { if } \beta=(i j, 1), \\ \{(2 i, 1),(2 j, 1)\}, & \text { if } \beta=(i j, 2) .\end{cases}
$$

Case 3. We then treat the case where $|\alpha \cap \beta|=2$.

Assume that $k=3$. Then $\beta=(1 i, 3)$ or $(2 i, 3)$, where $i \geqslant 4$. If $\beta=(1 i, 3)$, then $\Gamma(\alpha) \cap \Gamma(\beta)=\{(13, m),(2 i, 1) \mid 4 \leqslant m \leqslant n, m \neq i\}$, and thus $d(\alpha, \beta)=2$. Similarly, if $\beta=(2 i, 3)$, there exists $n-3$ paths of length 2 between $\alpha$ and $\beta$, and so $d(\alpha, \beta)=2$.

Suppose that $k \neq 3$. Then $\beta=(12, k),(1 i, 2),(2 i, 1),(13, k),(23, k),(3 i, 1),(3 i, 2)$, where $i, k \geqslant 4$. If $\beta=(12, k)$, then $\Gamma(\alpha) \cap \Gamma(\beta)=\{(1 m, 2),(2 m, 1) \mid 4 \leqslant m \leqslant n, m \neq k\}$, and so $d(\alpha, \beta)=2$. For these vertices $\beta=(1 i, 2),(2 i, 1),(13, k)$, or $(23, k)$, we have $\beta \in \Gamma(\alpha)$, and thus $d(\alpha, \beta)=1$. If $\beta=(3 i, 1), \Gamma(\alpha) \cap \Gamma(\beta)=\{(13, m),(23, i),(1 i, 2) \mid 4 \leqslant$ $m \leqslant n, m \neq i\}$, and hence $d(\alpha, \beta)=2$. Similarly, if $\beta=(3 i, 2), \Gamma(\alpha) \cap \Gamma(\beta)=$ $\{(23, m),(13, i),(2 i, 1) \mid 4 \leqslant m \leqslant n, m \neq i\}$, and so $d(\alpha, \beta)=2$.

Case 4. Let $|\alpha \cap \beta|=3$. Then $\beta=(23,1)$ or $(13,2)$.

For $\beta=(23,1)$, we have $d(\alpha, \beta)=2$ as $(13, m)$ is adjacent to both $\alpha$ and $\beta$, and thus, there are exactly $n-3$ paths of length 2 between $\alpha$ and $\beta$, where $m \neq 1,2,3$. Similarly, if $\beta=(13,2), d(\alpha, \beta)=2$ because there exist exactly $n-3$ paths $\alpha,(23, m), \beta$ of length 2 between $\alpha$ and $\beta$, where $m \neq 1,2,3$.

Thus, $\Gamma=\Gamma_{n}$ is of diameter 3 . 


\section{Quotients}

The action of $G=\operatorname{Sym}([n])$ on $V_{n}$ has three types of blocks as below

$$
\begin{aligned}
& B_{k}=\{(i j, k) \mid i, j \in[n] \backslash\{k\}\}, \text { size }\left(\begin{array}{c}
n-1 \\
2
\end{array}\right), \\
& B_{i j}=\{(i j, k) \mid k \in[n] \backslash\{i, j\}\}, \text { size } n-2, \\
& B_{i j k}=\{(i j, k),(j k, i),(k i, j)\}, \text { size } 3 .
\end{aligned}
$$

Let $\mathcal{B}_{1}=B_{k}^{G}, \mathcal{B}_{2}=B_{i j}^{G}$, and $\mathcal{B}_{3}=B_{i j k}^{G}$. Then $\left|\mathcal{B}_{1}\right|=n,\left|\mathcal{B}_{2}\right|=\left(\begin{array}{l}n \\ 2\end{array}\right)$, and $\left|\mathcal{B}_{3}\right|=\left(\begin{array}{c}n \\ 3\end{array}\right)$.

Lemma 7. If $n \geqslant 7$, then $V=V_{n}$ has exactly three non-trivial $G$-invariant partitions: $\mathcal{B}_{1}, \mathcal{B}_{2}$ and $\mathcal{B}_{3}$.

Proof. For the vertex $\beta=(23,1)$, the stabilizer $G_{\beta}=\operatorname{Sym}(\{2,3\}) \times \operatorname{Sym}([n] \backslash\{1,2,3\})$, and $G_{\beta}$ is contained in $G_{B_{1}}, G_{B_{23}}$ and $G_{B_{123}}$. Moreover, these three subgroups are maximal in $G$ and are the only proper subgroups of $G$ which properly contain $G_{\beta}$. Thus, $\mathcal{B}_{1}, \mathcal{B}_{2}$ and $\mathcal{B}_{3}$ are the only block systems of $G$ acting on $V_{n}$.

By Lemma 7 , we have three block systems $\mathcal{B}_{i}$ with $i=1,2$ or 3 , and we have three quotient graph $\Gamma_{\mathcal{B}_{i}}$. Clearly, the induced action of $G$ on $\mathcal{B}_{i}$ is equivalent to the action of $G$ on $[n]^{\{i\}}$, where $i=1,2$ or 3 . We thus identify $\mathcal{B}_{1}$ with $[n], \mathcal{B}_{2}$ with $[n]^{\{2\}}$, and $\mathcal{B}_{3}$ with $[n]^{\{3\}}$. The quotient graph $\Gamma_{\mathcal{B}_{1}}=\mathbf{K}_{n}=\mathbf{J}(n, 1)$. For $\Gamma_{\mathcal{B}_{2}}$, two vertices $B_{i j}$ and $B_{i^{\prime} j^{\prime}}$ are adjacent if and only if $\left|\{i, j\} \cap\left\{i^{\prime}, j^{\prime}\right\}\right|=1$, and so $\Gamma_{\mathcal{B}_{1}}=\mathbf{J}(n, 2)$. For $\Gamma_{\mathcal{B}_{3}}$, two vertices $B_{i j k}$ and $B_{i^{\prime} j^{\prime} k^{\prime}}$ are adjacent if and only if $\left|\{i, j, k\} \cap\left\{i^{\prime}, j^{\prime}, k^{\prime}\right\}\right|=2$. Thus, we have the following lemma.

Lemma 8. The quotient graph $\Gamma_{\mathcal{B}_{i}}$ is the Johnson graph $\mathbf{J}(n, i)$, where $i=1,2$ or 3 .

For a quotient graph $\Gamma_{\mathcal{B}}$, let $B, B^{\prime} \in \mathcal{B}$ be adjacent in $\Gamma_{\mathcal{B}}$. The induced subgraph $\left[B \cup B^{\prime}\right]$ of $\Gamma$ over $B \cup B^{\prime}$ is the graph with vertex set $B \cup B^{\prime}$ and edge set $E_{0}=\{\{u, v\} \in$ $\left.E \mid u, v \in B \cup B^{\prime}\right\}$. Then $\left[B \cup B^{\prime}\right]$ is a bipartite graph with biparts $B$ and $B^{\prime}$; denoted by $\left[B, B^{\prime}\right]$. We next determine the induced subgraph $\left[B, B^{\prime}\right]$ for the quotient graph $\Gamma_{\mathcal{B}_{i}}$.

For a graph $\Sigma=(V, E)$, the vertex-edge incidence graph is the bipartite graph with biparts $V$ and $E$ such that two vertices $v \in V$ and $w \in E$ are adjacent if and only if $v, w$ are incident in $\Sigma$. This incidence graph is also called the subdivision of $\Sigma$.

Lemma 9. Let $B, B^{\prime} \in \mathcal{B}_{1}$ be adjacent in $\Gamma_{\mathcal{B}_{1}}$. Then the induced subgraph $\left[B, B^{\prime}\right]$ consists of 2 copies of the subdivision of $\mathbf{K}_{n-2}$.

Proof. Since $(23,1) \in B_{1}$ is adjacent to $(34,2) \in B_{2}$, the vertices $B_{1}$ and $B_{2}$ are adjacent in the quotient $\Gamma_{\mathcal{B}_{1}}$. The edges of the induced subgraph $\left[B_{1}, B_{2}\right]$ are

$$
\{\{(2 i, 1),(i j, 2)\} \mid 3 \leqslant i \leqslant n, j \neq i, 1,2\} \cup\{\{(1 i, 2),(i j, 1)\} \mid 3 \leqslant i \leqslant n, j \neq i, 1,2\},
$$

which form two copies of the subdivision of $\mathbf{K}_{n-2}$.

A star $\mathbf{K}_{1, m}$ is a bipartite graph with $m+1$ vertices, in which there is one vertex that is adjacent to all other $m$ vertices. In particular, $\mathbf{K}_{1,2}$ is a path of length 2 . 
Lemma 10. Let $B, B^{\prime} \in \mathcal{B}_{2}$ be adjacent in $\Gamma_{\mathcal{B}_{2}}$. Then the induced subgraph $\left[B, B^{\prime}\right]$ consists of 2 copies of the star $\mathbf{K}_{1, n-3}$.

Proof. Since $(12,3) \in B_{12}$ is adjacent to $(23,4) \in B_{23}$, the vertices $B_{12}$ and $B_{23}$ are adjacent in the quotient $\Gamma_{\mathcal{B}_{2}}$. The edges of the induced subgraph $\left[B_{12}, B_{23}\right]$ are $\{\{(12,3)$, $(23, i)\} \mid i \geqslant 4\} \cup\{\{(23,1),(12, j)\} \mid j \geqslant 4\}$, which form two stars $\mathbf{K}_{1, n-3}$.

Lemma 11. Let $B, B^{\prime} \in \mathcal{B}_{3}$ be adjacent in $\Gamma_{\mathcal{B}_{3}}$. Then the induced subgraph $\left[B, B^{\prime}\right]$ consists of 2 paths of length 2 .

Proof. For the blocks $B=\{(12,3),(23,1),(31,2)\}$ and $B^{\prime}=\{(12,4),(24,1),(41,2)\}$, the induced subgraph $\left[B, B^{\prime}\right]$ has 4 edges

$$
\{(12,3),(14,2)\},\{(12,3),(24,1)\},\{(12,4),(13,2)\},\{(12,4),(32,1)\} .
$$

These edges form two paths of length 2:

$$
(23,1),(12,4),(13,2) \text {, and }(24,1),(12,3),(14,2) \text {. }
$$

Thus, $\left[B, B^{\prime}\right]=2 \mathbf{K}_{1,2}$.

\section{The automorphism group}

In this section, we determine the automorphism group Aut $\Gamma$.

Lemma 12. Let $n \geqslant 7$, and let $X$ be a subgroup such that $G \leqslant X \leqslant$ Aut $\Gamma$. Then $X$ is almost simple, and if $X \neq G$, then $X$ is primitive on $V$.

Proof. Let $M$ be a minimal normal subgroup of $X$. Suppose that $M$ is intransitive on $V$. Let $\mathcal{B}$ be the set of $M$-orbits on $V$. Then $\mathcal{B}$ is $X$-invariant and $G$-invariant. By Lemma 7 , $\mathcal{B}=\mathcal{B}_{i}$ with $i=1,2$ or 3 . For $B_{i} \in \mathcal{B}_{i}$, we have that $G_{B_{1}}^{B_{1}}=\mathrm{S}_{n-1}, G_{B_{2}}^{B_{2}}=\mathrm{S}_{n-2}$, and $G_{B_{3}}^{B_{3}}=\mathrm{S}_{3}$. Thus, $G_{B_{i}}^{B_{i}}$ is primitive, and so is $X_{B_{i}}^{B_{i}}$.

Let $K=X_{(\mathcal{B})}$, the kernel of $X$ acting on $\mathcal{B}$. Suppose that $K \neq 1$. Then $1 \neq K^{B} \triangleleft X_{B}^{B}$, and so $K^{B}$ is transitive as $X_{B}^{B}$ is primitive. Let $B^{\prime} \in \mathcal{B}$ be adjacent in $\Gamma_{\mathcal{B}}$ to $B$. Then $K$ is transitive on $B^{\prime}$, and since $|B|=\left|B^{\prime}\right|$, we conclude that the induced subgraph $\left[B, B^{\prime}\right]$ is regular, which is a contradiction by Lemmas $9-11$. Thus, $K=1$, and so $M=1$, which is a contradiction. So $M$ is transitive on $V$, and $X$ is quasiprimitive on $V$. Further, $M$ is non-abelian since $|V|=3\left(\begin{array}{l}n \\ 3\end{array}\right)$.

Now let $M=T_{1} \times T_{2} \times \ldots \times T_{l}$, where $l \geqslant 1$, and $T_{1} \cong T_{2} \cong \ldots \cong T_{l}$ are non-abelian simple groups. Then $M \cap G \triangleleft G$, and so $M \cap G=1, \mathrm{~A}_{n}$ or $\mathrm{S}_{n}$.

Suppose that $M \cap G=1$. Let $Z=M G=M: G$. If $Z$ is imprimitive on $V$, then a block system $\mathcal{B}=\mathcal{B}_{1}, \mathcal{B}_{2}$ or $\mathcal{B}_{3}$. Hence $Z \cong Z^{\mathcal{B}} \leqslant G^{\mathcal{B}} \cong \mathrm{S}_{n}$, which is not possible. Thus, $Z$ is primitive on $V$, and $G$ does not centralize $M$. By O'Nan-Scott's theorem (see [7]), we have that $l \geqslant n$, and $\frac{n(n-1)(n-2)}{2}=|V|=m^{l}$ or $m^{l-1}$ where $m \geqslant 5$, which is not possible.

Therefore, $M \cap G=\mathrm{A}_{n}$ or $\mathrm{S}_{n}$, and letting $L=\operatorname{soc}(G)=\mathrm{A}_{n}$, we have $L \leqslant M$. Since $L$ is non-abelian simple, $L$ is contained in a simple group $T_{i}$, say $T_{1}$. Hence, $T_{1}$ is transitive 
on $V$. If $l \geqslant 2$, then as $T_{2}$ centralizes $T_{1}$, we have that $T_{2}$ is semiregular on $V$. Then $|V|$ divides $\left|T_{1}\right|$, and $\left|T_{2}\right|=\left|T_{1}\right|$ divides $|V|$. So $T_{1}$ is regular on $V$, and since $G$ is transitive on $V, L \leqslant T_{1}$ is semiregular with at most 2 orbits. Since $T_{1}$ has no subgroup of index 2 and $G / L \cong \mathbb{Z}_{2}$, we have that $L=T_{1}$ is regular on $V$, which is a contradiction. Thus, $M=T_{1}$ is simple and $L \leqslant M$. Assume that there exists another minimal normal subgroup $N$ of $X$ such that $N \neq M$. Then $M \cap N=1$; however, the above argument with $N$ in the place of $M$ shows that $L \leqslant N$. So $M \cap N \geqslant L$, which is a contradiction. Therefore, $M$ is simple and the unique minimal normal subgroup of $X$, and hence $X$ is almost simple.

Suppose that $X>G$ and $X$ is imprimitive on $V$. Let $\mathcal{B}$ be a block system for $X$ on $V$. Then $\mathcal{B}$ is a block system for $G$ on $V$. By Lemma $7, \mathcal{B}=\mathcal{B}_{i}$ where $i=1,2$ or 3 , and by Lemma $8, \Gamma_{\mathcal{B}}=\mathbf{J}(n, i)$. As noticed in the Introduction, Aut $\Gamma_{\mathcal{B}}=\mathrm{S}_{n}$, and so $\mathrm{S}_{n} \cong G<X \cong X^{\mathcal{B}} \leqslant$ Aut $\Gamma_{\mathcal{B}} \cong \mathrm{S}_{n}$, which is not possible. Hence either $X=G$, or $X$ is primitive on $V$, as claimed.

A transitive permutation group $G$ on $\Omega$ is called $k$-homogeneous if $G$ is transitive on the set of $k$-subsets of $\Omega$, where $k$ is a positive integer.

Lemma 13. If $n \geqslant 8$, then Aut $\Gamma=G=\operatorname{Sym}([n])$.

Proof. Let $n \geqslant 8$. Suppose that $G<\operatorname{Aut} \Gamma$. Let $L \leqslant \operatorname{Aut} \Gamma$ be such that $G$ is a maximal subgroup of $L$. Since $G$ is transitive on $V$, the almost simple group $L$ has a factorization $L=G L_{\alpha}$. Further, since $L$ is primitive on $V$, the factorization $L=G L_{\alpha}$ is a maximal factorization. Thus, the triple $\left(L, G, L_{\alpha}\right)$ is classified in [11], see the MAIN THEOREM on page 1. An inspection of the candidates with one factor being $G=\mathrm{S}_{n}$, we conclude that one of the following holds:

(i) $n \leqslant 12$, or

(ii) $L=\mathrm{S}_{n+1}$, or

(iii) $L=\mathrm{S}_{m}$ or $\mathrm{A}_{m}$, and $G$ is $k$-homogenous of degree $m$, where $1 \leqslant k \leqslant 5$.

Consider the small groups where $n \leqslant 12$. We note that as $\Gamma$ is not a complete group, $L<\operatorname{Sym}(V)$.

Let $n=12$ first. Then $|V|=\frac{n(n-1)(n-2)}{2}=660$, and $L$ is a primitive group of degree 660. Hence $L$ lies in Appendix B of [7], which shows that $\operatorname{soc}(L)=\operatorname{PSL}(2,659)$ or $\operatorname{PSL}(2,11) \times \operatorname{PSL}(2,11)$. So $L$ does not contains $\mathrm{S}_{12}$, which is a contradiction. Similarly, the cases where $n=8,9$ and 10 are excluded.

Suppose that $n=11$. Then $|V|=495$, and $\Gamma$ is of valency 32. By Appendix B of [7], as $\mathrm{S}_{11}<L$, we conclude that $\left(L, L_{\alpha}\right)=\left(\mathrm{S}_{12}, \mathrm{~S}_{8} \times \mathrm{S}_{4}\right)$ or $\left(\mathrm{O}_{10}^{-}(2), 2^{8}: \mathrm{O}_{8}^{-}(2)\right)$. The former is not possible since $\mathrm{S}_{12} \neq \mathrm{S}_{11}\left(\mathrm{~S}_{8} \times \mathrm{S}_{4}\right)$, and the latter is not possible since $\mathrm{O}_{8}^{-}(2)$ does not have a transitive representation of degree at most 32 .

Next, let $L=\mathrm{S}_{n+1}$, with $n \geqslant 13$. Since $L=G L_{\alpha}$, the stabilizer $L_{\alpha}$ is a transitive permutation group of degree $n+1$ such that $\left|L: L_{\alpha}\right|=|V|=\frac{n(n-1)(n-2)}{2}$. Assume that $L_{\alpha}$ is primitive of degree $n+1$. By Bochert's theorem (see [21, Theorem 14.2]), 
$\left|L: L_{\alpha}\right| \geqslant\left[\frac{n+2}{2}\right]$ !. Computation shows that $n \leqslant 10$, which is a contradiction. Thus, $L_{\alpha}$ is imprimitive of degree $n+1$. Then $L_{\alpha}=\mathrm{S}_{b} \succ \mathrm{S}_{m}$, where $b m=n+1$, and thus,

$$
\frac{n(n-1)(n-2)}{2}=|V|=\left|L: L_{\alpha}\right|=\frac{(n+1) !}{(b !)^{l} m !}
$$

which is not possible.

Finally, assume that $G=\mathrm{S}_{n}$ is $k$-homogenous of degree $m$, and $L=\mathrm{S}_{m}$ or $\mathrm{A}_{m}$, and $L_{\alpha}=\mathrm{S}_{k} \times \mathrm{S}_{m-k}$, where $k \leqslant 5$. Since $L$ is not 2-transitive on $V$, we have $k \geqslant 2$. Thus, by the classification of 2 -homogeneous groups, we conclude that $n \leqslant 8$, which is a contradiction.

Therefore, Aut $\Gamma=G=\operatorname{Sym}([n])$, as claimed.

\section{Proof of Theorem 2}

In this section, we prove the main theorem.

By Lemma 6, part (i) of Theorem 2 is true. By Lemma 8, Theorem 2 (ii) holds.

For $n=4$ or 5 , Theorem 2 is proved by Lemma 5 . Thus, we next assume $n \geqslant 6$.

For $n=6$ or 7 , a computation using Gap shows that Aut $\Gamma_{n}=\mathrm{S}_{n}$, and for $n \geqslant 8$, Lemma 13 shows that Aut $\Gamma=G$. Then, by Lemma 3, $\Gamma$ is half-transitive, as in part (iii).

Finally, assume that $\Gamma$ is a Cayley graph of a group $R$. Then $R$ is regular on $V$ (see [3, Proposition 16.3]), and hence $R$ is 3-homogeneous but not 3 -transitive on $[n]$. Further, as $|R|=|V|=3\left(\begin{array}{l}n \\ 3\end{array}\right), R$ is not sharply 3-homogeneous on $[n]$. Inspecting 3homogeneous groups which are not 3 -transitive, refer to [7, Theorem $9.4 \mathrm{~B}]$, we conclude that $R=\operatorname{A\Gamma L}(1,8)$ or $\operatorname{PSL}(2, q)$ where $q \equiv 3(\bmod 4)$. So $n=8$ or $q+1$, respectively. This proves part (iv) of Theorem 2 .

\section{Acknowledgements}

The authors are grateful to the referee for the valuable comments.

\section{References}

[1] B. Alspach, D. Marušič, and L. Nowitz. Constructing graphs which are 1/2-transitive. J. Austral. Math. Soc. Ser. A, 56:391-402, 1994.

[2] B. Alspach and M. Y. Xu. 1/2-transitive graphs of order 3p. J. Algebraic Combin., 3:347-355, 1994.

[3] N. Biggs. Algebraic Graph Theory. Cambridge University Press, New York, 1992.

[4] I. Z. Bouwer. Vertex and edge-transitive but not 1-transitive graphs. Canad. Math. Bull., 13:231-237, 1970. 
[5] A. E. Brouwer, A. M. Cohen and A. Neumaier. Distance-Regular Graphs. Ergeb. Math. Grenzgeb. (Results in Mathematics and Related Areas (3)), vol. 18, SpringerVerlag, Berlin, 1989.

[6] J. H. Conway, R. T. Curtis, S. P. Norton, R. A. Parker, and R. A. Wilson. Atlas of Finite Groups. Oxford Univ. Press, London/New York, 1985.

[7] J. D. Dixon and B. Mortimer. Permutation Groups. Springer, 1991.

[8] Y. Q. Feng, K. S. Wang and C. X. Zhou. Tetravalent half-transitive graphs of order 4p. Europ. J. Combin., 28:726-733, 2007.

[9] G. A. Jones. Automorphisms and regular embeddings of merged Johnson graphs. European J. Combin., 26(3-4):417-435, 2005.

[10] C. H. Li and H. S. Sim. On half-transitive metacirculant graphs of prime-power order. J. Combin. Theory Ser. B, 81:45-57, 2001.

[11] M. Liebeck, C. E. Praeger and J. Saxl. The maximal factorizations of the finite simple groups and their automorphism groups. Mem. Amer. Math. Soc., 86(432), 1990.

[12] D. Marušič. Recent developments in half-transitive graphs. Discrete Math., 182:219231, 1998.

[13] D. Marušič and C. E. Praeger. Tetravalent graphs admitting half-transitive groups actions: alternating cycles. J. Combin. Theory Ser. B, 75:185-205, 1999.

[14] D. Marušič and P. Šparl. On quartic half-arc-transitive metacirculants. J. Algebraic Combin., 28:365-395, 2008.

[15] Mark Ramras and Elizabeth Donovan. The Automorphism Group of a Johnson Graph. Siam J. Discrete Math., 25:267-270, 2011.

[16] M. Šajna. Half-transitivity of some metacirculants. Discrete Math., 185:117-136, 1998.

[17] Shu-Jiao Song, C. H. Li, and Dian-Jun Wang. Classifying a family of edge-transitive metacirculant graphs. J. Algebraic Combin., 35:497-513, 2012.

[18] Primoz Sparl. A classification of tightly attached half-arc-transitive graphs of valency 4. J. Combin. Theory Ser. B, 98, 2008.

[19] Primoz Sparl. Almost all quartic half-arc-transitive weak metacirculants of Class II are of Class IV. Discrete Math., 310:1737-1742, 2010.

[20] W. T. Tutte. Connectivity in Graphs. University of Toronto Press, Toronto, 1966.

[21] H. Wielandt. Finite Permutations Groups. Academic Press Inc, 1964.

[22] M. Y. Xu. Half-transitive graphs of prime-cube order. J. Algebraic Combin., 1:275$282,1992$. 